Published in final edited form as:

Clin Genitourin Cancer. 2019 April ; 17(2): e273-e280. doi:10.1016/j.clgc.2018.11.006.

\title{
Stereotactic Radiosurgery for Multiple Brain Metastases From Renal-Cell Carcinoma
}

\author{
Zabi Wardak $^{1,2}$, Alana Christie ${ }^{1,4}$, Alex Bowman ${ }^{1,3}$, Strahinja Stojadinovic $^{2}$, Lucien Nedzi ${ }^{2}$, \\ Sam Barnett ${ }^{5}$, Toral Patel ${ }^{5}$, Bruce Mickey ${ }^{1,5}$, Tony Whitworth ${ }^{1,5}$, Raquibul Hannan ${ }^{1,2}$, James \\ Brugarolas $^{1,3}$, and Robert Timmerman ${ }^{1,2}$ \\ ${ }^{1}$ Kidney Cancer Program, Simmons Comprehensive Cancer Center \\ 2Department of Radiation Oncology \\ ${ }^{3}$ Division of Hematology and Oncology, Department of Internal Medicine \\ ${ }^{4}$ Department of Internal Medicine \\ ${ }^{5}$ Department of Neurological Surgery, UT Southwestern Medical Center, Dallas, TX
}

\begin{abstract}
Treatment with radiosurgery prevents neurocognitive decline associated with whole-brain radiotherapy. After review of data of 38 patients treated with radiosurgery for brain metastases from metastatic renal cell carcinoma, the local control at 1 year was $92 \%$. There was no difference in survival on the basis of number of brain metastases. With therapeutic advancements, a greater number of patients might be candidates for radiosurgery.
\end{abstract}

Background: Brain metastases (BM) pose a significant problem in patients with metastatic renal-cell carcinoma (mRCC). Local and systemic therapies including stereotactic radiosurgery (SRS) are rapidly evolving, necessitating reassessments of outcomes for modern patient management.

Patients and Methods: The mRCC patients with BM treated with SRS were reviewed. Patient demographics, clinical history, and SRS treatment parameters were identified.

Results: Among 268 patients with mRCC treated between 2006 and 2015, 38 patients were identified with BM. A total of $243 \mathrm{BM}$ were treated with SRS with 1 to $26 \mathrm{BMs}$ treated per SRS session (median, 2 BMs). The median (range) BM size was $0.6(0.2-3.1) \mathrm{cm}$ and median (range) SRS treatment dose was 18 (12-24) Gy. Treated BM local control rates at 1 and 2 years were 91.8\% (95\% confidence interval, 85.7-95.4) and 86.1\% (95\% confidence interval, 77.1-91.7), respectively. BM control declined for larger tumors. Survival after 1-year was 57.5\% (95\% CI 40.2-71.4) for all patients. Survival was not statistically different between patients with $<5 \mathrm{BM}$ versus $\geq 5$ BM. Survival was prognostic based on International Metastatic Renal Cell Carcinoma

\footnotetext{
Address for correspondence: Zabi Wardak, MD, Kidney Cancer Program, Simmons Comprehensive Cancer Center, UT Southwestern Medical Center Department of Radiation Oncology, 2280 Inwood Rd, Dallas, TX 73590, Zabi.wardak@utsouthwestern.edu.

Disclosure

The authors have stated that they have no conflict of interest.

Supplemental Data

A supplemental figure accompanying this article can be found in the online version at https://doi.org/10.1016/j.clgc.2018.11.006.
} 
Database (IMDC) risk groups in patients with $<5 \mathrm{BM}$. Two patients experienced grade 3 radiation necrosis requiring surgical intervention.

Conclusion: SRS is effective in controlling BM in patients with mRCC. Over half of treated patients survive past a year, and no differences in survival were noted in patients with $>5$ metastases. Prognostic risk categories based on systemic disease (IMDC) are predictive of survival in this BM population, with limited rates of symptomatic radiation necrosis.

\section{Keywords}

CNS; Kidney cancer; Radiation; SRS

\section{Introduction}

Current estimates state approximately 170,000 cases of brain metastases (BM) are diagnosed per year, and among patients with metastatic renal-cell carcinoma (mRCC), the incidence of $\mathrm{BM}$ is as high as $15 \% .^{1,2}$ Thanks to improvements in local and systemic therapy for extracranial disease, patients with $\mathrm{mRCC}$ are living longer and may be at a greater likelihood of developing BM. ${ }^{3-5}$ Furthermore, continued improvements in imaging, with increasing magnetic resonance imaging (MRI) magnet strength and new imaging protocols, have increased the sensitivity and specificity of the detection of intracranial metastases. 6,7

The efficacy of systemic therapy alone in the treatment of renal-cell carcinoma (RCC) BM is limited, with a prospective clinical trial with sunitinib in untreated BM resulting in no objective responses. ${ }^{8}$ Data with respect to the efficacy of other targeted therapies and mTORC1 inhibitors are nonconclusive, so some form of local therapy is standard. ${ }^{9}$ Surgical resection of BM is associated with an improved survival over whole-brain radiotherapy (WBRT) alone. However, for patients with a greater intracranial or systemic disease burden, or for patients who are not candidates for resection, stereotactic radiosurgery (SRS) offers an alternative option with excellent control rates. ${ }^{10}$

The treatment of intracranial metastases with radiotherapy has historically involved WBRT. That paradigm has shifted in recent years, with clinical trials showing that compared to WBRT plus SRS, SRS alone has lower rates of neurocognitive decline without a detriment in overall survival. ${ }^{11,12}$ Historical candidacy for SRS has been based on the number of intracranial metastases; however, technological improvements in both treatment planning software and treatment delivery instruments permit the treatment of a greater intracranial disease burden. ${ }^{13,14}$

In this study we sought to determine the outcomes of patients treated with RCC with BM using SRS in the modern era, including the efficacy of treating multiple metastases.

\section{Patients and Methods}

Patients with a diagnosis of RCC between the years 2006 and 2015 were screened for the presence of $\mathrm{BM}$ at either initial presentation of metastatic disease or were evaluated during their clinical course. All patients treated with SRS for BM between the years 2008 and 2015 comprised the study cohort, as that was the earliest date of record availability of radiation 
planning. All patients were treated with a model 4C Leksell gamma knife or Perfexion. Patients were eligible for SRS on the basis of evaluation by the treating physicians, with no limitation on the number of metastases treated. Patient demographics, risk group, histology, staging, number of SRS sessions, number of metastases treated, size of treated metastases in both maximum dimension and volume, dose delivered, prescription isodose line, and anatomic location were reviewed.

On the day of SRS, patients received local anesthetic and moderate sedation with placement of the Leksell Frame G. A stereotactic planning MRI was performed with axial 3dimensional T1 fat-suppressed postcontrast images. The planning MRI was imported into the GammaPlan software suite with target delineation, and planning was performed by the treating radiation oncologist and neurosurgeon. Patients were followed radiographically at regular intervals (typically 2- to 3-month intervals) after SRS with MRI imaging with contrast (or computed tomography with contrast if unable to undergo MRI). Progression of treated metastases was defined by Response Evaluation Criteria in Solid Tumors 1.1.

Descriptive statistics are provided as frequencies and percentages for categorical variables, and as medians and ranges for continuous variables. For lesions that were imaged after gamma knife treatment, proportional hazards models were built to analyze the risk of failure of local control. Kaplan-Meier curves were also produced to visualize local control over time, as well as overall survival after diagnosis of BM. All analyses were completed at the . 05 significance level by SAS 9.4 software (SAS Institute, Cary, NC).

\section{Results}

A total of 268 patients were identified with $\mathrm{mRCC}$ at our institution, of whom 76 (28.4\%) were identified as having a diagnosis of BM between 2006 and 2015. Of these 76 patients, 56 were identified at diagnosis or while receiving first-line systemic therapy. Furthermore, 38 of these patients underwent SRS for 243 BM at our institution and are the subject of this report. This study includes 5 additional patients that did not have longitudinal relationship with medical oncology and were excluded from the companion study published by Bowman et al (Clin GU Cancer). The median (range) age of patients was 65 (28-78) years (Table 1). The most common histology was clear-cell RCC, and 74\% of patients underwent only one gamma knife session, with a median number of lesions treated of 2 per session (Table 2). The most common intracranial location for metastases was the frontal lobe (39\% of lesions), followed by the parietal and temporal lobes (15\% of lesions). The median (range) size of treated metastases was $0.6(0.2-3.1) \mathrm{cm}$. The median (range) dose of treated metastases was 18 (12-24) Gy prescribed to a 50\% (40-80\%) isodose line (Table 2). The prescription dose was typically chosen on the basis of the size of the treated BM (eg, $24 \mathrm{~Gy}$ for lesions $<2 \mathrm{~cm}$, $18 \mathrm{~Gy}$ for lesions 2 to $3 \mathrm{~cm}$, and $15 \mathrm{~Gy}$ for lesions $>3 \mathrm{~cm}$ ).

A total of 177 metastases in 29 patients had radiographic follow-up, with 9 patients lost to follow-up after SRS. The median (range) follow-up was $12(0-80)$ months. There were no local failures within 6 months. The local control of all treated lesions at 1 year was $91.8 \%$ (95\% confidence interval [CI], 85.7-95.4), and the rate at 2 years was $86.1 \%$ (95\% CI, 77.191.7). A significant association was found between local control based on lesion size, with 
metastases $<2 \mathrm{~cm}$ significantly more likely to be controlled than metastases $>2 \mathrm{~cm}(P<$. 0001) (Figure 1). There also was a significant association between local control and metastases volume, with lesions $<2 \mathrm{cc}$ more likely to be controlled than those $>2 \mathrm{cc}(P<$. 0001). There was no association with local control with either dose delivered $(P=.52)$ or isodose line prescribed $(P=.33)$ (Table 3$)$.

The median overall survival after diagnosis of BM was 13.8 months (95\% CI, 8.1-28.0), and the 1-year survival was $57.5 \%$ (95\% CI, 40.2-71.4). The 1-year overall survival after a diagnosis of BM for patients with a single BM was 56.3\% (95\% CI, 29.5-76.2) and for patients with multiple BM was $59.1 \%$ (95\% CI, 36.1-76.2). For patients with 1 to $4 \mathrm{BM}$, the 1-year survival was53.1\% (95\% CI, 33.2-69.5), and for patients with 5 or more lesions, the 1-year survival was 70.0\% (95\% CI, 32.9-89.2) (Figure 2). These differences among patients with few or many lesions were not significant.

Three patients had toxicity related to the treated BM by SRS. One patient had increased edema and enhancement of 2 adjacent left frontal metastases with dose overlap, measuring 1.2 and $0.9 \mathrm{~cm}$ in size with both treated with $24 \mathrm{~Gy}$ each at the $50 \%$ isodose line (Figure 3). This patient initiated therapy with bevacizumab with clinical improvement and ultimately underwent resection of the metastases, which pathologically identified viable tumor; however, the adjacent dose overlap contributed to a component of necrosis as well. A second patient had edema after SRS to a frontal and occipital metastasis, measuring 2.9 and $2.3 \mathrm{~cm}$ in size each treated with $15 \mathrm{~Gy}$ at the $50 \%$ isodose line, requiring prolonged dexamethasone therapy. The third patient was treated initially in 2011 for a $2.9 \mathrm{~cm}$ right temporal metastasis to a dose of $15 \mathrm{~Gy}$ at the $50 \%$ isodose line which progressed, and he underwent irradiation again 2 years later, in 2013 , to $15 \mathrm{~Gy}$ at the $50 \%$ isodose line. Three months after that, he experienced seizures. The patient initially opted for treatment with hyperbaric oxygen without relief and ultimately underwent resection 1 year after repeat irradiation, with pathology returning results of $40 \%$ seemingly viable tumor and $60 \%$ radiation necrosis.

When reviewing outcomes of patients on the basis of International Metastatic Renal Cell Carcinoma Database (IMDC) risk group, there was no significant difference in BM control according to IMDC risk group $(P=.24)$. There was a significant difference in survival of patients with identified unfavorable risk compared to favorable or intermediate with fewer than 4 metastases $(P=.0001)$. For patients with 5 or more metastases, there was a nonsignificant decreased survival-again in patients in the unfavorable risk group compared to those in the favorable and intermediate risk groups (Supplemental Figure 1 in the online version).

\section{Discussion}

$\mathrm{BM}$ represent the most common intracranial tumor, with a reported incidence of approximately $15 \%$ in patients with RCC. ${ }^{1,2,15}$ In our cohort, the incidence of BM was found to be greater, at $28 \%$. With improvements in local and systemic therapy, patients are living longer. As a result, there is an increasing likelihood of developing multiple BM over the course of their care. ${ }^{3-5}$ 
Most patients facing BM would prefer to avoid central nervous system toxicity, particularly toxicities that affect quality of life. In this context, SRS is an attractive treatment option. SRS has several advantages over both WBRT and surgical resection for BM. It avoids the global adverse effects of WBRT, including fatigue and neurocognitive decline. Radiosurgery can be performed in patients with comorbidities who are not surgical candidates, can treat metastases in anatomic locations that are not amenable to resection, and is a minimally invasive outpatient procedure with no significant recovery time, allowing patients to initiate or continue systemic therapy sooner without waiting for surgical wound healing. It also spares patients from undergoing multiple invasive surgical procedures if they continue to develop intracranial metastases over their clinical course.

Treatment of intracranial metastases has historically involved WBRT. However, this paradigm has changed thanks to several recent trials that compared WBRT plus SRS to SRS alone. These trials showed lower rates of neurocognitive decline without a detriment in overall survival. Improvements in radiation planning have led to the development of hippocampal-sparing WBRT, and its use with memantine has been shown to result in less of a neurocognitive decline compared to traditional WBRT. ${ }^{16}$ However, WBRT is more commonly foregone in favor of SRS alone with close surveillance as the treatment of choice for preservation of cognitive function. ${ }^{17-19}$ In these trials, candidacy for SRS has been limited to patients with fewer than 4 metastases. More recent evidence suggests that the use of SRS alone may be appropriate in patients with 5 to 10 metastases. A study from Japan showed that the median and overall survival of patients treated with SRS alone was similar if patients had 2 to 4 metastases or 5 to 10 metastases. ${ }^{20}$ An international consortium of institutions reviewed 711 patients with BM from RCC between 2006 and 2016. They found that the utilization of WBRT has declined from $50 \%$ to $22 \%$, with an increased utilization of SRS from $48 \%$ to $58 \%$ and a nonsignificant improvement in survival compared to WBRT. ${ }^{15}$ A second series from the National Cancer Data Base of patients receiving cranial radiotherapy between 2004 and 2014 for RCC found that $35 \%$ of patients received SRS, with an increasing trend from $27 \%$ in 2005 to $40 \%$ in 2014. In this series, there was found to be a significantly improved survival in patients who received SRS versus non-SRS cranial radiotherapy. ${ }^{21}$ In our series, we routinely treated patients with multiple metastases, even up to 26 total lesions, without toxicity, reflecting the transition away from WBRT in favor of SRS.

In patients with a solitary symptomatic BM who are deemed as having medically operable lesions, we consider this option as a priority, particularly for those with symptomatic mass effect. Surgery may be particularly appropriate for patients with a long disease-free interval and otherwise good prognosis. However, in patients who are unfit or unwilling, or if the lesion is not amenable to resection, SRS can be used as an effective method for local control. It has been shown across multiple histologies to have high rates of local control, and our experience has shown that for RCC, the 1-year local control rate is $>90 \%$, which is comparable to other series of SRS for RCC BM. ${ }^{22-24}$ Our experience with stereotactic body radiotherapy for extracranial disease has also shown a 1-year local control of $>90 \%$, which suggests that aggressive local therapy to all sites of disease with stereotactic radiotherapy, when there are limited metastatic sites, can be highly efficacious. ${ }^{25}$ 
Our data reveal that smaller central nervous system metastases, both in size and volume, are more likely to be durably controlled, thus highlighting the efficacy of higher doses for smaller metastases but the need for improving control of larger metastases. Current dosing for larger metastases is based on the maximum dimension of the metastasis. ${ }^{18}$ Historical dose selection is based on a phase 1 Radiation Therapy Oncology Group study that did not reach the maximum tolerated dose for these larger tumors. In addition, those studies combined WBRT with SRS, thus possibly limiting the maximum achievable SRS dose when delivered alone. At our institution, we are conducting a phase 1 study using SRS without WBRT that, we hope, will permit higher tolerable dosing, which might improve control of larger lesions (ClinicalTrials.gov NCT03508752). Alternatively, fractionating these larger metastases in hopes of delivering a greater radiobiologic dose may be useful to maximize tumor control probability. With the improvements in SRS delivery (eg, now with frameless treatment on the latest Icon gamma knife), expansion of patient eligibility for fractionation for larger metastases will become easier to perform.

Two of the most commonly used prognostic models for mRCC include the Memorial Sloan Kettering Cancer Center model and the IMDC risk group. The IMDC risk group is defined by 6 factors: performance status, time from diagnosis to treatment, hemoglobin concentration, calcium concentration, neutrophil count, and platelet count. ${ }^{26}$ Elsewhere we show that these IMDC-defined risk groups also affect outcomes in patients with BM. Specifically in patients presenting with BM in a poor risk group, the hazard ratio for death was 1.99 (95\% CI, 0.997-3.96; $P=.0462)$.

In our experience, a greater number of patients were identified with limited intracranial disease burden; however, patients with 5 or more BM were not shown to have a worse survival than patients with a lesser burden of intracranial disease, even when considering IMDC risk group. This is in comparison to other series that have shown a worse survival with 5 or more BM; our findings may be the result of a smaller cohort of patients. ${ }^{15}$ Nevertheless, these patients should be considered for SRS in hopes of maintaining their quality of life and preventing neurocognitive decline. The Icon gamma knifee- and linear accelerator-based treatment platforms (eg, Elements by Brainlab) can be used to efficiently treat a greater burden of intracranial disease, and the treatment of metastases can be distributed over several days so that overlap of adjacent lesions can be minimized so as to limit the risk of radiation necrosis. With adjacent metastases, a greater volume of normal brain will receive an intermediate radiation dose if treated simultaneously, with an increasing volume receiving $>12$ Gy shown to be a predictor of increasing risk of symptomatic radiation necrosis. ${ }^{27}$ This was visualized in one patient in our cohort, highlighting the need to consider dose overlap with metastases adjacent to one another. With these considerations, and thanks to the improvements in the technology, the ability to provide neurocognitivesparing, noninvasive therapy to a greater number of patients with BM with RCC will become a realistic opportunity.

\section{Conclusion}

SRS for BM from RCC is highly effective, with > 90\% 1-year local control rate and durability of control to 2 years. The treatment is most efficacious for smaller metastases; 
improvements such as escalated dosing or fractionation are needed for treating larger lesions. Patients with multiple BM (> 5 lesions) from RCC did not have a worse survival and should be considered for SRS to achieve intracranial tumor control while avoiding neurologic decline.

\section{Supplementary Material}

Refer to Web version on PubMed Central for supplementary material.

\section{Acknowledgment}

J.B. is supported by National Institutes of Health grant P50CA196516.

\section{References}

1. Wyler L, Napoli CU, Ingold B, et al. Brain metastasis in renal cancer patients: metastatic pattern, tumour-associated macrophages and chemokine/chemoreceptor expression. Br J Cancer 2014; 110:686-94. [PubMed: 24327013]

2. Bianchi M, Sun M, Jeldres C, et al. Distribution of metastatic sites in renal cell carcinoma: a population-based analysis. Ann Oncol 2012; 23:973-80. [PubMed: 21890909]

3. Hammers HJ, Plimack ER, Infante JR, et al. Safety and efficacy of nivolumab in combination with ipilimumab in metastatic renal cell carcinoma: the CheckMate 016 study. J Clin Oncol 2017; 35:3851-8. [PubMed: 28678668]

4. Choueiri TK, Escudier B, Powles T, et al. Cabozantinib versus everolimus in advanced renal-cell carcinoma. N Engl J Med 2015; 373:1814-23. [PubMed: 26406150]

5. Motzer RJ, Escudier B, McDermott DF, et al. Nivolumab versus everolimus in advanced renal-cell carcinoma. N Engl J Med 2015; 373:1803-13. [PubMed: 26406148]

6. Biswas J, Nelson CB, Runge VM, et al. Brain tumor enhancement in magnetic resonance imaging: comparison of signal-to-noise ratio (SNR) and contrast-to-noise ratio (CNR) at 1.5 versus 3 tesla. Invest Radiol 2005; 40:792-7. [PubMed: 16304483]

7. Ba-Ssalamah A, Nobauer-Huhmann IM, Pinker K, et al. Effect of contrast dose and field strength in the magnetic resonance detection of brain metastases. Invest Radiol 2003; 38:415-22. [PubMed: 12821855]

8. Chevreau C, Ravaud A, Escudier B, et al. A phase II trial of sunitinib in patients with renal cell cancer and untreated brain metastases. Clin Genitourin Cancer 2014; 12:50-4. [PubMed: 24268852]

9. Maria B, Antonella V, Michela R, et al. Multimodality treatment of brain metastases from renal cell carcinoma in the era of targeted therapy. Ther Adv Med Oncol 2016; 8:450-9. [PubMed: 27800033]

10. Du Y, Pahernik S, Hadaschik B, et al. Impact of resection and systemic therapy on the survival of patients with brain metastasis of metastatic renal cell carcinoma. J Neurooncol 2016; 130:221-8. [PubMed: 27538910]

11. Chang EL, Wefel JS, Hess KR, et al. Neurocognition in patients with brain metastases treated with radiosurgery or radiosurgery plus whole-brain irradiation: a randomised controlled trial. Lancet Oncol 2009; 10:1037-44. [PubMed: 19801201]

12. Brown PD, Jaeckle K, Ballman KV, et al. Effect of radiosurgery alone vs. radio-surgery with whole brain radiation therapy on cognitive function in patients with 1 to 3 brain metastases: a randomized clinical trial. JAMA 2016; 316:401-9. [PubMed: 27458945]

13. Wang JZ, Rice R, Mundt AJ, et al. Feasibility and advantages of using flattening filter-free mode for radiosurgery of multiple brain lesions. Pract Radiat Oncol 2012; 2:e165-71. [PubMed: 24674180]

14. Liu H, Andrews DW, Evans JJ, et al. Plan quality and treatment efficiency for radiosurgery to multiple brain metastases: non-coplanar RapidArc vs. gamma knife. Front Oncol 2016; 6:26. [PubMed: 26904504] 
15. Sperduto PW, Deegan BJ, Li J, et al. Effect of targeted therapies on prognostic factors, patterns of care, and survival in patients with renal cell carcinoma and brain metastases. Int J Radiat Oncol Biol Phys 2018; 101:845-53. [PubMed: 29976497]

16. Gondi V, Pugh SL, Tome WA, et al. Preservation of memory with conformal avoidance of the hippocampal neural stem-cell compartment during whole-brain radiotherapy for brain metastases (RTOG 0933): a phase II multi-institutional trial. J Clin Oncol 2014; 32:3810-6. [PubMed: 25349290]

17. Aoyama H, Shirato H, Tago M, et al. Stereotactic radiosurgery plus whole-brain radiation therapy vs. stereotactic radiosurgery alone for treatment of brain metastases: a randomized controlled trial. JAMA 2006; 295:2483-91. [PubMed: 16757720]

18. Andrews DW, Scott CB, Sperduto PW, et al. Whole brain radiation therapy with or without stereotactic radiosurgery boost for patients with one to three brain metastases: phase III results of the RTOG 9508 randomised trial. Lancet 2004; 363:1665-72. [PubMed: 15158627]

19. Kocher M, Soffietti R, Abacioglu U, et al. Adjuvant whole-brain radiotherapy versus observation after radiosurgery or surgical resection of one to three cerebral metastases: results of the EORTC 22952-26001 study. J Clin Oncol 2011; 29:134-41. [PubMed: 21041710]

20. Yamamoto M, Serizawa T, Shuto T, et al. Stereotactic radiosurgery for patients with multiple brain metastases (JLGK0901): a multi-institutional prospective observational study. Lancet Oncol 2014; 15:387-95. [PubMed: 24621620]

21. Haque W, Verma V, Butler EB, et al. Utilization of stereotactic radiosurgery for renal cell carcinoma brain metastases. Clin Genitourin Cancer 2018; 16: e935-43. [PubMed: 29680768]

22. Mori Y, Kondziolka D, Flickinger JC, et al. Stereotactic radiosurgery for brain metastasis from renal cell carcinoma. Cancer 1998; 83:344-53. [PubMed: 9669818]

23. Muacevic A, Kreth FW, Mack A, et al. Stereotactic radiosurgery without radiation therapy providing high local tumor control of multiple brain metastases from renal cell carcinoma. Minim Invasive Neurosurg 2004; 47:203-8. [PubMed: 15346315]

24. Amendola BE, Wolf AL, Coy SR, et al. Brain metastases in renal cell carcinoma: management with gamma knife radiosurgery. Cancer J 2000; 6:372-6. [PubMed: 11131486]

25. Wang CJ, Christie A, Lin MH, et al. Safety and efficacy of stereotactic ablative radiation therapy for renal cell carcinoma extracranial metastases. Int J Radiat Oncol Biol Phys 2017; 98:91-100. [PubMed: 28587057]

26. Heng DY, Xie W, Regan MM, et al. External validation and comparison with other models of the International Metastatic Renal-Cell Carcinoma Database Consortium prognostic model: a population-based study. Lancet Oncol 2013; 14:141-8. [PubMed: 23312463]

27. Korytko T, Radivoyevitch T, Colussi V, et al. 12 Gy gamma knife radiosurgical volume is a predictor for radiation necrosis in non-AVM intracranial tumors. Int J Radiat Oncol Biol Phys 2006; 64:419-24. [PubMed: 16226848] 


\section{Clinical Practice Points}

- $\quad$ SRS is increasingly being utilized for BM to prevent neurocognitive decline.

- The outcomes of patients with BM from mRCC have been modernized to reflect this change in practice, showing excellent local control rates.

- $\quad$ SRS should be offered for patients with a greater number of BM beyond historical inclusion criteria.

- With improvements in systemic therapy, patients with mRCC live longer and will be spared the neurocognitive decline of WBRT. 


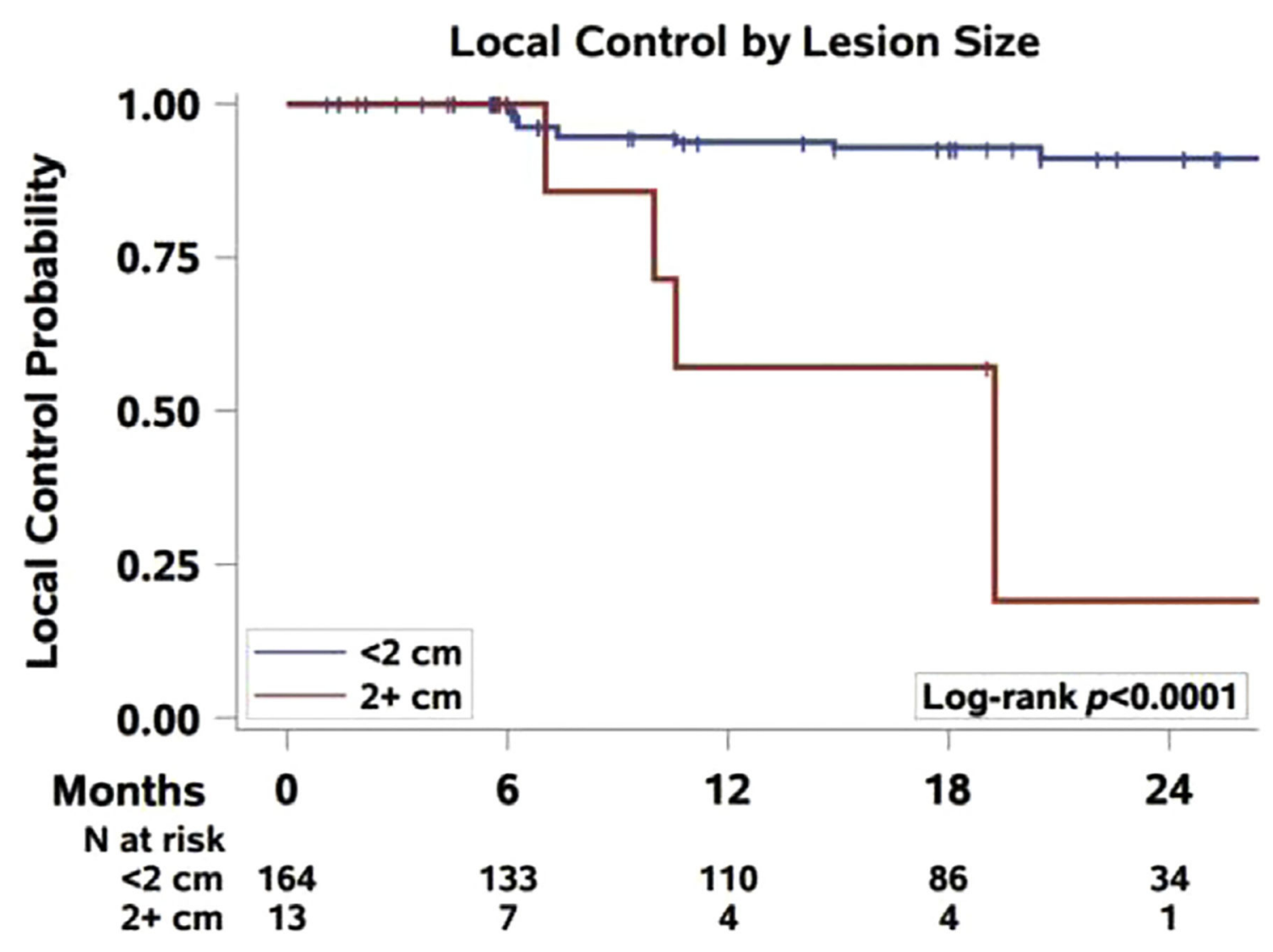

Figure 1.

Local Control of Brain Metastasis by Lesion Size 

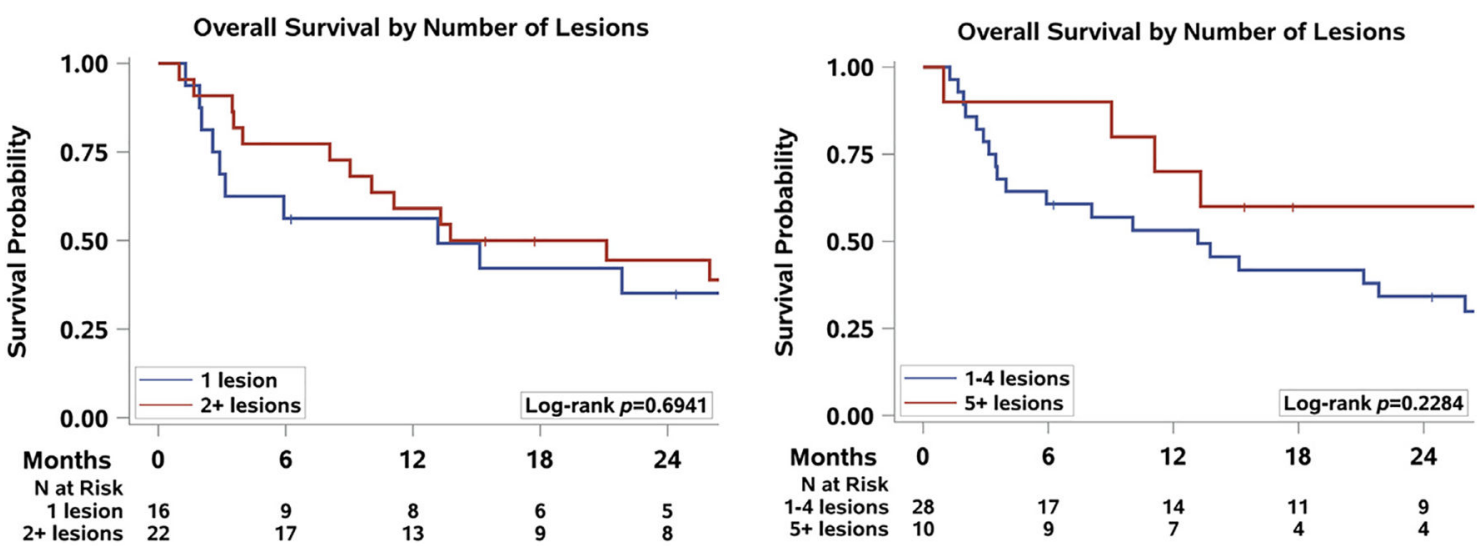

Figure 2.

Overall Survival by Number of Lesions 


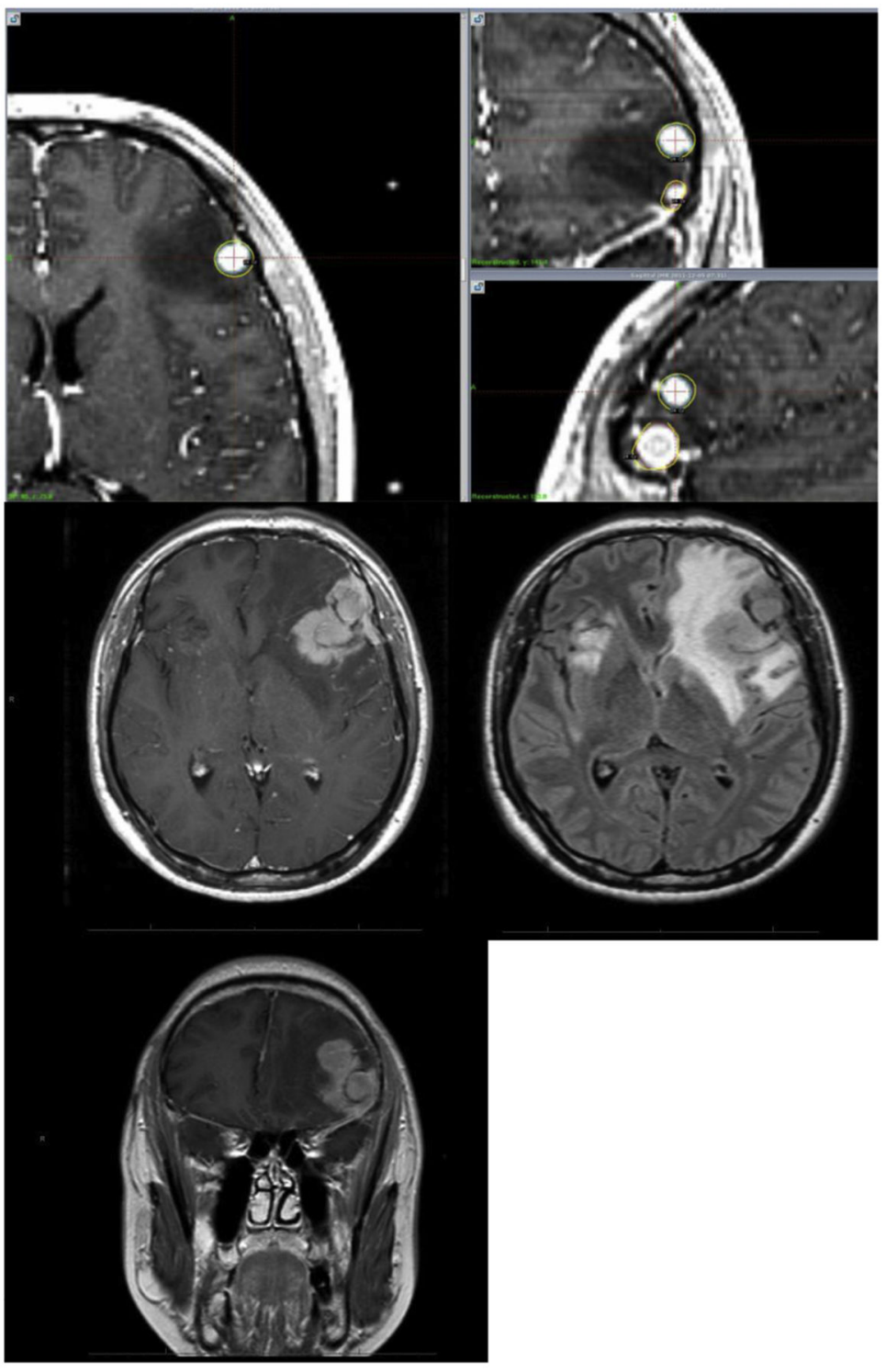

Figure 3.

Radiation Necrosis of Adjacent Metastases Treated in Single Session. Top Row, SRS Treatment Plan With Isodose Line in Yellow; Bottom Rows, Posttreatment Radiation Necrosis on T1 Postaxial/Coronal Imaging and Treatment Edema on FLAIR Imaging Abbreviations: FLAIR = fluid-attenuated inversion recovery; SRS = stereotactic radiosurgery. 


\section{Table 1}

Characteristics of 38 Patients

\begin{tabular}{|c|c|}
\hline Characteristic & Value \\
\hline Age (years), median (range) & $65.4(28.4-78.3)$ \\
\hline \multicolumn{2}{|l|}{ Gender } \\
\hline Female & $13(34.2)$ \\
\hline Male & $25(65.8)$ \\
\hline \multicolumn{2}{|l|}{ IMDC Risk Group } \\
\hline Favorable (0) & $7(18.4)$ \\
\hline Intermediate (1-2) & $20(52.6)$ \\
\hline Unfavorable (3-6) & $7(18.4)$ \\
\hline Missing & $4(10.5)$ \\
\hline \multicolumn{2}{|l|}{ Histology } \\
\hline $\mathrm{ccRCC}$ & $33(86.8)$ \\
\hline Non-ccRCC & $3(7.9)$ \\
\hline Missing & $2(5.3)$ \\
\hline \multicolumn{2}{|l|}{ pT (At Presentation) } \\
\hline 1 & $4(10.5)$ \\
\hline 2 & $4(10.5)$ \\
\hline 3 & $19(50)$ \\
\hline 4 & $4(10.5)$ \\
\hline Missing & $7(18.4)$ \\
\hline \multicolumn{2}{|l|}{ pN } \\
\hline $\mathrm{X}$ & $14(36.8)$ \\
\hline 0 & $10(26.3)$ \\
\hline 1 & $14(36.8)$ \\
\hline \multicolumn{2}{|l|}{$\mathbf{M}$} \\
\hline 0 & $17(44.7)$ \\
\hline 1 & $21(55.3)$ \\
\hline \multicolumn{2}{|l|}{ No. of Gamma Knife Therapy Courses } \\
\hline 1 & $28(73.7)$ \\
\hline 2 & $2(5.3)$ \\
\hline 3 & $4(10.5)$ \\
\hline 4 & $4(10.5)$ \\
\hline \multicolumn{2}{|l|}{ Lesions } \\
\hline Total no. of lesions treated, median (IQR) & $3(1-8)$ \\
\hline Total size of lesions $(\mathrm{cm})$, median (IQR) & $3.0(1.2-7.6)$ \\
\hline Total volume of lesions (cc), median (IQR) & $2.4(0.5-6.9)$ \\
\hline
\end{tabular}

Data are presented as $\mathrm{n}(\%)$ unless otherwise indicated.

Abbreviations: $\mathrm{cc}=$ clear cell; IMDC = International Metastatic Renal Cell Carcinoma Database Consortium; IQR = interquartile range; $\mathrm{RCC}=$ renal-cell carcinoma. 


\section{Table 2}

Stereotactic Radiosurgery Treatment Parameters

\begin{tabular}{l|c} 
Characteristic & Value \\
No. of lesions treated in course ( $\mathrm{n}=60$ courses) & $2(1-26)$ \\
Total sum of size of lesions in course (cm) & $2.1(0.3-17.3)$ \\
Total volume of lesions in course (cc) & $1.0(0.01-17.2)$ \\
Lesions (N = 243 Lesions) & \\
Size (cm) & $0.6(0.2-3.1)$ \\
Volume (cc) & $0.1(0.01-11.6)$ \\
Therapy dose (Gy) & $18(12-24)$ \\
Isodose of treatment $(\%)$ & $50(40-80)$ \\
Lesion Location & \\
Cerebellar & $31(12.8 \%)$ \\
Deep & $11(4.5 \%)$ \\
Frontal & $95(39.1 \%)$ \\
Insula + Temporal & $42(17.3 \%)$ \\
Occipital & $23(9.5 \%)$ \\
Parietal & $37(15.2 \%)$ \\
Other & $4(1.6 \%)$ \\
\hline
\end{tabular}

Data are presented as $\mathrm{n}(\%)$ or median (range). 


\section{Table 3}

\section{Brain Metastasis Local Control in 177 Lesions}

\begin{tabular}{l|c|c} 
Characteristic & HR $(\mathbf{9 5} \% \mathbf{C I})$ & $\boldsymbol{P}$ \\
Lesion Size & & \\
$<1 \mathrm{~cm}$ & Reference & .0008 \\
$1-2 \mathrm{~cm}$ & $1.14(0.35,3.68)$ & \\
$>2 \mathrm{~cm}$ & $7.52(2.56,22.10)$ & \\
Lesion Volume & & \\
$<1 \mathrm{cc}$ & Reference & .0015 \\
$1-2 \mathrm{cc}$ & $-{ }^{a}$ & \\
$>2 \mathrm{cc}$ & $6.57(2.36,18.31)$ & \\
Treatment Dose & & \\
$12-18$ Gy & Reference & .5205 \\
$20-24$ Gy & $0.71(0.25,2.04)$ & \\
Treatment Isodose & & \\
$40-50 \%$ & Reference & .3390 \\
$55-80 \%$ & $0.60(0.21,1.70)$ & \\
\hline
\end{tabular}

Abbreviations: $\mathrm{CI}=$ confidence interval; $\mathrm{HR}=$ hazard ratio

${ }^{a}$ No events occurred in group. 